\title{
Experimental Study of Inclusive Muon Spectra from Electron-Positron Collisions in the Energy Region $33 \leqslant \sqrt{s} \leqslant 38.54 \mathrm{GeV}$
}

B. Adeva, D. P. Barber, U. Becker, J. Berdugo, G. Berghoff, A. Boehm, J. G. Branson, J. D. Burger, M. Capell, M. Cerrada, C. C. Chang, H. S. Chen, M. Chen, M. L. Chen, M. Y. Chen, Y. S. Chu, R. Clare, E. Deffur, M. Demarteau, P. Duinker, Z. Y. Feng, H. S. Fesefeldt, D. Fong, M. Fukushima, D. Harting, T. Hebbeker, G. Herten, M. C. Ho, M. M. Ilyas, D. Z. Jiang, W. Krenz, P. Kuijer, Q. Z. Li, D. Luckey, E. J. Luit, C. Mana, M. A. Marquina, G. G. G. Massaro, R. Mount, H. Newman, M. Pohl, F. P. Poschmann, R. R. Rau, J. P. Revol, S. Rodriguez, M. Rohde, J. A. Rubio, H. Rykaczewski, J. Salicio, I. Schulz, K. Sinram, M. Steuer, G. M. Swider, H. W. Tang, D. Teuchert, Samuel C. C. Ting, K. L. Tung, M. Q. Wang, M. White, H. G. Wu, S. X. Wu, B. Wyslouch, B. Zhou, R. Y. Zhu, and Y. C. Zhu III. Physikalisches Institut, Technische Hochschule, D-5100 Aachen, Federal Republic of Germany, and Deutsches Elektronen Synchrotron DESY, D-2000 Hamburg 52, Federal Republic of Germany, and Laboratory for Nuclear Science, Massachusetts Institute of Technology, Cambridge, Massachusetts 02139, and Junta de Energia Nuclear, Madrid, Spain, and Nationaal Instituut voor Kernfysica en Hoge-Energiefysica, 1009-DB Amsterdam, The Netherlands, and Institute of High Energy Physics, Chinese Academy of Science, Beijing, People's Republic of China, and California Institute of Technology, Pasadena, California 91125

(Received 22 April 1983)

The results of a high-statistics study of inclusive muon spectra at PETRA are reported. Improved mass limits have been obtained for heavy quarks, heavy leptons, and charged Higgs particles. It is shown that the fragmentation properties of $b$ quarks and $c$ quarks are different, with the mean fragmentation variables $\left\langle z_{b}\right\rangle=0.75 \pm 0.03 \pm 0.06,\left\langle z_{c}\right\rangle=0.46$ $\pm 0.02 \pm 0.05$ and the average semileptonic branching ratio for the $B$ and $C$ hadrons $R(B)$ $=(10.5 \pm 1.5 \pm 1.3) \%, R(C)=(11.5 \pm 1.0 \pm 1.7) \%$.

PACS numbers: $13.10 .+q, 13.65 .+\mathrm{i}, 14.80 . \mathrm{Dq}$

In the last twenty years experimental studies of leptons from synchrotrons and colliders have yielded important information in our understanding of particle physics. The discovery of the $J$ $(c \bar{c}),{ }^{1}$ the $\Upsilon(b \bar{b}),{ }^{2}$ and the heavy lepton $\tau^{3}$ are good examples. It follows naturally that we can use the study of leptons at higher energies to search for new quark states, ${ }^{4}$ new heavy leptons $\left(L^{ \pm}\right),{ }^{5}$ and charged Higgs particles $\left(H^{ \pm}\right){ }^{6}$ Fur ther, these studies can provide information on the semileptonic branching ratios and fragmentation functions of known heavy quarks.

New heavy leptons and charged Higgs particles would be pair produced in $e^{+} e^{-}$. The heavy lepton should have a branching ratio into $\mu \bar{\nu}_{\mu} \nu_{L}$ of about $14 \%$, and into $\nu_{L}$ +hadrons of about $60 \%$. The charged Higgs particle is expected to decay dominantly into $\tau \bar{\nu}_{\tau}$ and $s \bar{c}$ (or $b \bar{c}$ ), with the relative branching ratios of these modes not clearly predicted. Both types of new particles will then give rise to events with an isolated muon roughly opposite to a hadron jet. In the case of the charged Higgs particle, the probability of producing such an event will depend upon the relative branching ratio into $\tau \nu_{\tau}$.

The production mechanism for hadron events containing muons,

$$
e^{+} e^{-} \rightarrow \operatorname{jet}_{1}(\mu)+\text { jet }_{2},
$$

can be visualized via the following sequence ${ }^{7}$ : (i) Heavy-quark pairs $Q \bar{Q}(Q=b, c$ quarks $)$ are produced via QED $e^{+} e^{-} \rightarrow Q \bar{Q}$. (ii) The fragmentation of $Q \rightarrow M+q$ produces hadron $M$, which carries the signature of $Q$, and $q=u, d, s$ quarks which fragment into more hadrons. (iii) The hadron $M$ decays semileptonically, $M \rightarrow \mu+\boldsymbol{x}$.

The hadron $M$ can be identified because its decay $\mu$ has a distinctive large transverse momentum (with respect to the jet axis), $P_{T} \sim m_{Q} / 4$. Since the production rates for the heavy quarks are known, a measurement of the rate of muons with transverse momenta in this region determines the semileptonic branching ratio of $M$. Further, the momentum of the $\mu$ along the jet direction provides information on the momentum carried by $M$ and thus on the fragmentation functions of the heavy quarks.

For the present studies we have used data from the MARK -J detector, a $4 \pi$ calorimeter which measures the directions and energies of $e, \mu, \gamma$, and jets. ${ }^{8}$ We collected a total of $76 \mathrm{pb}^{-1}$ integrated luminosity in the region $33 \leqslant \sqrt{s} \leqslant 38.54$ 
GeV. From 25000 hadron events we select inclusive muon events with the criteria described in detail earlier. ${ }^{8}$

The inclusive muon sample contains 806 events with an isolated muon opposite to a hadron jet, dominantly due to $\tau$ pair production. By searching for events where the muon and the hadron jet are acoplanar $\left(>30^{\circ}\right)$, we unambiguously limit the mass of a new sequential heavy lepton to be greater than $18 \mathrm{GeV}$. The increase in this limit over that reported previously ${ }^{5}$ is mainly due to a recent increase in the PETRA beam energy. Our limit ${ }^{6}$ on the mass of a charged Higgs particle, assuming that its branching ratio into $\tau \nu_{\tau}$ is greater than $25 \%$, is slightly improved to $M_{H^{ \pm}}$ $>14 \mathrm{GeV}$.

To obtain mass limits on the production of new quarks and to study the fragmentation processes, we select ${ }^{8}$ the sample of hadron events containing muons in one of the jets, and reduce the effect of hard-gluon emission by collecting only those events with a broad jet oblateness $O_{b}<0.3$. After cuts to reduce the background from hadron punchthrough, we are left with 850 events. The remaining punchthrough (13\%) and decay (19\%) backgrounds are then statistically subtracted from the data. The punchthrough background has been simulated by a Monte Carlo calculation. We reject most of this background by requiring that the muon make a smooth series of chamber hits in two dimensions as it passes through the iron spectrometer. The punchthrough Monte Carlo calculation agrees with the number of rejected events. The efficiency for real muons to pass these cuts has been tested with our data sample on $e^{+} e^{-} \rightarrow e^{+} e^{-} \mu^{+} \mu^{-}$. Background from $\pi$ and $K$ decay is accurately computed in the Monte Carlo program. We include in our systematic errors (quoted below) $50 \%$ uncertainty in the punchthrough background and $20 \%$ in the decay background. The background $P_{T}$ spectrum peaks $300 \mathrm{MeV}$ lower than that from charm and the $P_{\|}$spectrum is peaked at much lower values than that of charm or bottom.

The Monte Carlo calculation includes radiative corrections, detector simulation, and the assumption that $B$ decays via $B \rightarrow C+x$ only. A spectator model $^{9}$ of the $B$-meson decay was used and the mass of the final charm system was varied between 1.9 and $2.4 \mathrm{GeV}$, given an average $C(D$ meson) momentum in the $B$ rest frame approximately $1.6 \mathrm{GeV} / c$. The rms resolution $\Delta P_{T} / P_{T}$ is about 0.5 , due to a momentum resolution $\Delta P / P$ $\sim 0.25$ and the error in the reconstruction of the jet axis.

In the framework of perturbative $\mathrm{QCD}$, we use a simple Feynman-Field ${ }^{7}$ model of fragmentation. Let $E_{M}$ and $E_{Q}$ be the energy of $M$ and $Q$ and $P_{M}$ and $P_{Q}$ be their momentum along the $Q$ direction. The quantity $z \equiv\left(E_{M}+\boldsymbol{P}_{M}\right) /\left(E_{Q}+P_{Q}\right)$ is a meas ure of the energy which the heavy quark retains after fragmentation. On the basis of the uncertainty principle ${ }^{10}$ the fragmentation function for $Q \rightarrow M+q$ can be parametrized ${ }^{11}$ in terms of $h_{Q}$ as

$$
f_{Q}(z)=\frac{1}{z}\left(1-\frac{1}{z}-\frac{h_{Q}^{2}}{1-z}\right)^{-2} \text {. }
$$

Let $B$ and $C$ be the hadronic states with $b$ and $c$ quark signatures, respectively, and let $R(B)$ and $R(C)$ be their average semileptonic-decay branching ratios. Figure 1(a) shows the differential cross section in $P_{T}^{2}$ compared with the pion distribution. ${ }^{12}$ The muon $P_{T}^{2}$ distribution is distinctly different from the pion distribution, with a shoulder in the range $2 \lesssim P_{T}^{2} \lesssim 5 \mathrm{GeV}^{2}$ which is indicative of the decay of a particle of mass around $5 \mathrm{GeV}$. Figure 1 (b) shows the data compared with the Monte Carlo predictions for the total $\mu$ rate and its components $B \rightarrow \mu+x$ and $C$ $\rightarrow \mu+x$. We have grouped the small $B \rightarrow C \rightarrow \mu+x$ contribution into the $C \rightarrow \mu+x$ sample. The shoul-

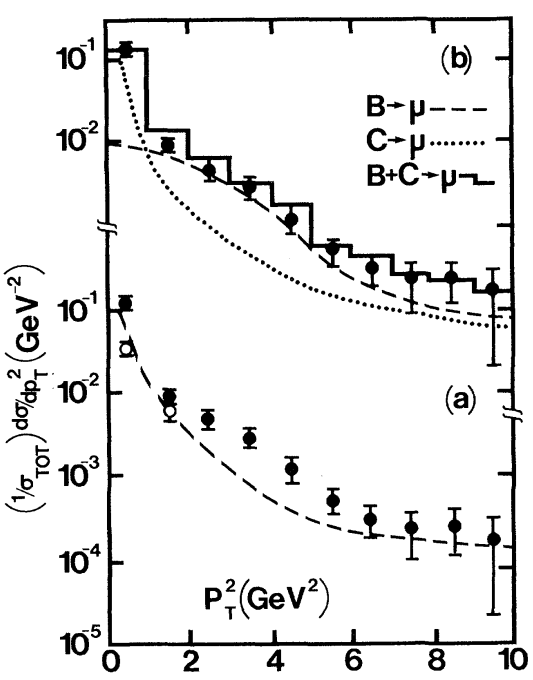

FIG. 1. (a) The $P_{T}{ }^{2}$ distributions of muons normalized to the total hadronic cross section $\sigma_{T}$. The open circles are the cross section with $P>2 \mathrm{GeV}$; the solid points are the cross section extrapolated to all $P$. They differ only in the first two points with $P_{T}{ }^{2}<2(\mathrm{GeV})^{2}$. The inclusive $\pi$ spectrum is also indicated as a dashed curve, scaled by $10^{-2}$. (b) Comparison of the data with Monte Carlo predictions, including individual $B \rightarrow \mu$ and $C \rightarrow \mu$ contributions. 


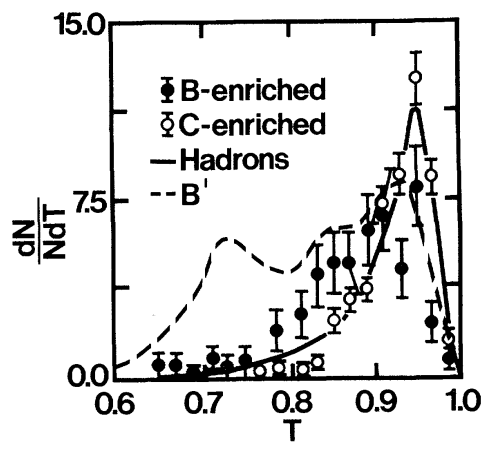

FIG. 2. The normalized thrust distribution of the $B$ - and $C$-enriched samples and all hadronic events. The dashed curve is the Monte Carlo prediction for the $B$-enriched sample with an additional charge- $-\frac{1}{3}$ quark $\left(b^{\prime}\right)$ of mass $16 \mathrm{GeV}$.

der is well explained by $B$ decays, and the Monte Carlo prediction agrees with the data. A $P_{T}$ cut is used to divide the data into $B$-enriched and $C$ enriched samples. The $B$-enriched sample $\left(\boldsymbol{P}_{\boldsymbol{T}}\right.$ $>1.2 \mathrm{GeV}$ ) has a composition, according to Monte Carlo calculations, of $64 \% \mathrm{~B}$ decays, while the expected composition of the $C$-enriched sample $\left(\psi_{T}<1.2 \mathrm{GeV}\right)$ is $85 \% C$ decays.

Figure 2 shows the thrust distribution for the $B$ - and $C$-enriched samples and all hadron events. The $B$-enriched sample exhibits a broader distribution than does the sample of hadrons. No significant difference exists between the $C$-enriched sample and the hadron sample. Similar to Fig. 1 the thrust distributions of the $B$-enriched and $C$-enriched samples can be explained by QCD with known quarks. The $P_{T}$ and thrust distributions enable us to obtain a limit on the mass of new charge $-\frac{1}{3}$ quarks $\left(b^{\prime}\right)$ of $m>16 \mathrm{GeV}$ (Fig. 2) ${ }^{4}$ and on charge $-\frac{2}{3}$ quarks $(t)$ of $m>19$ $\mathrm{GeV}^{8}$ Of course, $b^{\prime}$ and $t$ quarks with masses only slightly greater than the $b$ quark cannot be ruled out by this method alone. Their existence, however, is inconsistent with the agreement between our measured $B$ semileptonic branching ratio and that measured on the $\Upsilon(4 s) .^{13}$

Figures 3(a) and 3(b) show the $x=2 P / \sqrt{s}$ dis tributions for $B$-enriched and $C$-enriched samples, respectively, together with the Monte Carlo predictions. The $x$ distribution depends mainly on the fragmentation properties of the quarks. The fact that the average $x$ of the $B$ sample is significantly higher than that of the $C$ sample implies that $\left\langle z_{b}\right\rangle$ is much larger than $\left\langle z_{c}\right\rangle$.

As seen from Figs. 1 and 2 the $P_{T}$ and thrust distributions separate $B$ and $C$ samples independently. Figure 3 shows that the $x$ (or $P$ ) distribu-

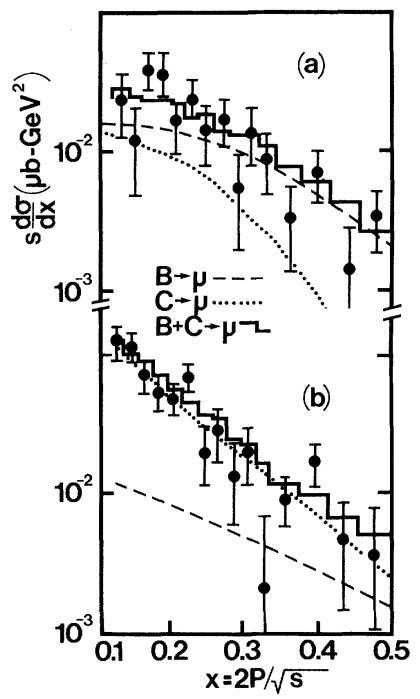

FIG. 3. The $x$ distributions for (a) the $B$-enriched sample, and (b) the $C$-enriched sample. The Monte Carlo curves are also shown.

tion is a measure of $\left\langle z_{b}\right\rangle$ and $\left\langle z_{c}\right\rangle$. To obtain the values of $R(B), R(C)$, and $h_{Q}$ we divide the data into an $8 \times 8 \times 8$ three-dimensional grid in the variables of $P$ and $P_{T}$ of the muon and the thrust. From the maximum-likelihood method we find fis-15 $^{13}$

$$
\begin{aligned}
& R(C)=(11.5 \pm 1.0 \pm 1.7) \%, \\
& R(B)=(10.5 \pm 1.5 \pm 1.3) \%, \\
& \left|h_{c}\right|=0.8 \pm 0.1 \pm 0.2, \\
& \left|h_{b}\right|=0.15 \pm 0.03 \pm 0.05 .
\end{aligned}
$$

This yields $\left\langle z_{c}\right\rangle=0.46 \pm 0.02 \pm 0.05$ and $\left\langle z_{b}\right\rangle$ $=0.75 \pm 0.03 \pm 0.06$. The ratio $\left|h_{c}\right| /\left|h_{b}\right|$ is 5.3 $\pm 1.3 \pm 2.2$, and may be compared with the ratio of the quark masses ${ }^{11} m_{b} / m_{c}$, which is approximately 3. The first errors ${ }^{16}$ are statistical. The second errors are systematic, including uncertainty in the background subtraction, the simulation of detector resolution and acceptance, the variation of mean transverse momenta of the primary particles $B$ and $C$ from 200 to $500 \mathrm{MeV}$, and the uncertainties in the decay $B \rightarrow \mu+\nu+(C$ $+x)$. Our value of $\left\langle z_{b}\right\rangle /\left\langle z_{c}\right\rangle \approx 1.6$ shows that $b$ and $c$ quarks have different fragmentation properties, with the $b$ quark retaining $75 \%$ of its energy in fragmentation.

The quantity $\left\langle z_{Q}\right\rangle$ may be determined without assumption of functional form by dividing the available $z$ region into many intervals and fitting the data bin by bin. With ten equal bins, this yields $\left\langle z_{b}\right\rangle=0.74 \pm 0.10,\left\langle z_{c}\right\rangle=0.46 \pm 0.05$. 
We thank Professor V. Soergel and Professor P. Soeding for their support. This work was supported in part by the Deutsches Bundesminister ium fur Forschung und Technologie.

${ }^{1} J$. J. Aubert et al., Phys. Rev. Lett. 33, 1404 (1974); J. E. Augustin et al., Phys. Rev. Lett. $\underline{33}, 1406$ (1974); J. M. Feller et al., Phys. Rev. Lett. 40, 274 (1978); R. H. Schindler et al., Phys. Rev. D 24, 78 (1981).

${ }^{2} \mathrm{~S}$. W. Herb et al., Phys. Rev. Lett. 39, 252 (1977); W. R. Innes, Phys. Rev. Lett. 39, 1240 (1977); C. Bebek et al., Phys. Rev. Lett. 46, 84 (1981); K. Chadwick, et al., Phys. Rev. Lett. $46, \overline{88}$ (1981); L. J. Spencer et al., Phys. Rev. Lett. $\overline{47}, 771$ (1981).

${ }^{3}$ M. L. Perl et al., Phys. Lett. 63B, 466 (1976).

${ }^{4} M$. Chen, in Proceedings of the Banff Summer Institute in Particles and Fields, Banff, Canada, August 1981 (unpublished); J. Buerger, in Proceedings of the International Symposium on Lepton and Photon Interactions, edited by W. Pfeil (Physikalisches Institut, Universität Bonn, Bonn, 1981), p. 115; P. Duinker, Rev. Mod. Phys. 54, 325 (1982).

${ }^{5}$ B. Adeva et al., Phys. Rev. Lett. $\underline{48}, 967$ (1982); D. P. Barber et al., Phys. Rev. Lett. 45, 1904 (1980); R. Brandelik et al., Phys. Lett. 99B, 163 (1981); Ch. Berger et al., Phys. Lett. 99B, 489 (1981).

${ }^{6}$ A. Ali et al., Nucl. Phys. B191, 93 (1981); B. Adeva et al., Phys. Lett. 115B, 345 (1982); W. Bartel et al., Phys. Lett. 114B, 211 (1982); H. J. Behrend et al., Phys. Lett. 114B, 287 (1982); R. Brandelik et al., Phys.
Lett. 122B, 95 (1983).

${ }^{7}$ R. D. Field and R. P. Feynman, Nucl. Phys. B136, 1-76 (1978).

${ }^{8}$ D. P. Barber et al., Phys. Rep. 63, 337 (1980).

${ }^{9}$ A. Ali et al., Phys. Lett. 93B, 155 (1980), and Nucl. Phys. B168, 409 (1980).

${ }^{10}$ M. Suzuki, Phys. Lett. 71B, 139 (1977); A. Ali, in Physics and Contemporary Needs (Plenum, New York, 1982), Vol. 5.

${ }^{11}$ C. Peterson et al., Phys. Rev. D 27, 105 (1983).

${ }^{12} \mathrm{R}$. Brandelik et al., Phys. Lett. 113 B, 98 (1982); G. Wolf, DESY Report No. 82-077, 1982 (unpublished).

${ }^{13}$ Our $R(B)$ can be compared with the value $(12.4 \pm 1.7$ $\pm 3.1) \%$ measured on the resonance $\Upsilon^{\prime \prime \prime}$. K. Chadwick et al., Phys. Rev. D 27, 473 (1983).

${ }^{14}$ The world average branching ratio is $19_{-3}^{+4} \%$ for $D^{ \pm} \rightarrow e+x$ and $<6 \%$ for $D^{0}$. M. Roos et al. (Particle Data Group), Phys. Lett. 111B, 1 (1982).

${ }^{15}$ For earlier measurements on $h_{c}$, see W. Bartel et al., DESY Report No. 80/86, 1980 (unpublished); K. Kleinknecht and B. Renk, Z. Phys. C 17, 325 (1983); D. Lueke et al., DESY Report No. 82/073, 1982 (unpublished). For preliminary results on $h_{b}$, see G. H. Trilling, J. Phys. (Paris), Colloq. 43, C3-58 (1982); J. M. Yelton et al., Phys. Rev. Lett. 49, 430 (1982); M. Althoff $e t$ al., DESY Report No. 83/010, 1983 (unpublished).

${ }^{16}$ These errors include the effects of correlations between the parameters and represent the $68 \%$ (onestandard-deviation) confidence interval in each parameter, with all other parameters free to vary. It should be noted that the probability distribution is approximately Gaussian in the $h$ 's, not in the more commonly quoted parameter $h^{2}$. 